\title{
A QUANTITATIVE ANALYSIS OF TUNNELING CURRENT IN A MOS CELL FOR A LOW-VOLTAGE MICROCONTROLLER
}

\author{
M. A. GRADO-CAFFARO and M. GRADO-CAFFARO
}

C/Julio. Palacios 11, 9으 28029-Madrid, Spain

(Received 28 May 1997; In final form 22 July 1997)

The tunneling current density in a MOS cell for a low-voltage microcontroller based on EEPROM is calculated for high electric strengths. Furthermore, this current density is discussed in terms of the oxide thickness and an approximate expression for the velocity of charge carriers is derived.

Keywords: Tunneling current; EEPROM; oxide thickness

\section{INTRODUCTION}

It is well-known the role played by the tunneling effect in certain electronic devices. In particular, MOS transistors involved in EEPROM for low-voltage microcontrollers exhibit various attractive features from the strict point of view of physical electronics. One of these features is related to the tunneling current of MOS cells; this current is very sensitive to the thickness of the involved oxide layer since the electric field in this layer is dependent upon this thickness and the current is strongly dependent on the electric-field strength. The aim of this paper consists of to discuss quantitatively some aspects related to the above dependences as well as carrier-velocity considerations. 


\section{THEORY}

Firstly we formulate the well-known expression for the above tunneling current density $[1,2]$ :

$$
J=\frac{e^{3} E^{2} m_{0}}{16 \pi^{2} \hbar E_{g}^{3 / 2} m_{n}^{*}} \exp \left(-\frac{4 \sqrt{2 m_{n}^{*} E_{g}^{3}}}{3 e \hbar E}\right)
$$

where $e$ is the electron charge, $E$ denotes strength of the electric field in the oxide layer of the $n$-channel MOS considered, $m_{0}$ is the electron rest-mass, $m_{n}^{*}$ is the tunneling electron effective mass, $\hbar$ is the reduced Planck's constant and $E_{g}$ is the barrier height of $\mathrm{Si}$ and $\mathrm{SiO}_{2}$ at cathode side (we consider an $\mathrm{Al}-\mathrm{SiO}_{2}-\mathrm{Si}$ device).

In addition, we want to remark that this MOS device has two gates: the control gate and the floating gate [3]. It has been claimed that when the drain voltage is reduced from $5 \mathrm{~V}$ to $3 \mathrm{~V}$, the corresponding charge pump output voltage also diminishes [3]. As a consequence, the control gate voltage becomes smaller; this means that the voltage drop across the tunnel oxide will decrease [3]. The diminished tunneling of electrons onto the floating gate gives rise to a slow erase operation resulting in EEPROM fallout [3].

Now we have $E=V_{\mathrm{ox}} / t_{\mathrm{ox}}$ where $V_{\mathrm{ox}}$ is the voltage drop across the tunnel oxide and $t_{\mathrm{ox}}$ is the oxide thickness. On the other hand, if $t_{\mathrm{ox}}$ is sufficiently small, $E$ can be regarded as a high electric strength so that we replace Eq. (1) by a few terms of its Taylor expansion around the origin; by taking into account also that $m_{n}^{*} \approx 1.1 m_{0}$, then Eq. (1) becomes

$$
J \approx \frac{e^{3} V_{\mathrm{ox}} E_{g}^{-3 / 2}}{16 \pi^{2} \hbar t_{\mathrm{ox}}}\left(\frac{V_{\mathrm{ox}}}{t_{\mathrm{ox}}}-\frac{4 \sqrt{2 m_{0} E_{g}^{3}}}{3 e \hbar}\right)
$$

Since $J=n e v$, where $n$ is the electron density and $v$ the velocity of electrons, then from Eq. (2) we get:

$$
v \approx \frac{e^{2} V_{\mathrm{ox}} E_{g}^{-3 / 2}}{16 \pi^{2} n \hbar t_{\mathrm{ox}}}\left(\frac{V_{\mathrm{ox}}}{t_{\mathrm{ox}}}-\frac{4 \sqrt{2 m_{0} E_{g}^{3}}}{3 e \hbar}\right)
$$




\section{CONCLUSIONS}

We have found Eqs. (2) and (3) in terms of various parameters; among them, we distinguish $t_{\mathrm{ox}}$ which, in practice, is $t_{o x} \approx 100 \AA[3,4]$. Notice that, for relatively small $t_{\mathrm{ox}}, v$ is relatively high (see Eq. (3)); this agrees with Ref. [3]. Finally, we want to remark that the above considerations are very relevant in the context of EEPROM cells [3,4].

\section{References}

[1] Sze, S. M. (1969). Physics of semiconductor devices (Wiley).

[2] Chen, I., Holland, S. and Hu, C. (1985). IEEE Trans. Electron Dev., ED-32, 413.

[3] Ku, J. W. and Chao, S. C. Semiconductor Int., May 1997, 105-110.

[4] Ajika, N., Ohi, M., Arima, H., Matsukawa, T. and Tsubouchi, N. (1990). IEDM Tech. Dig., 115. 

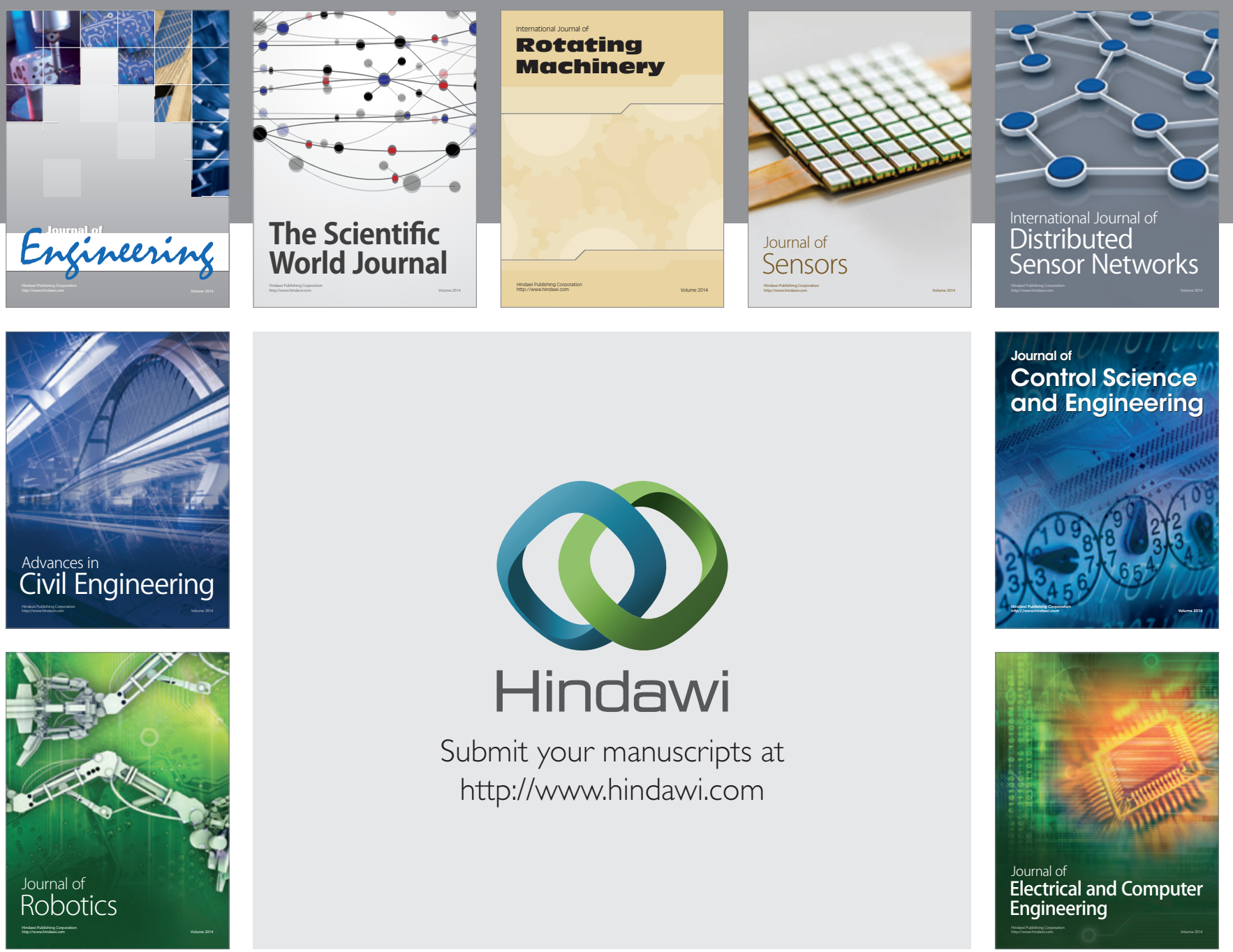

Submit your manuscripts at

http://www.hindawi.com
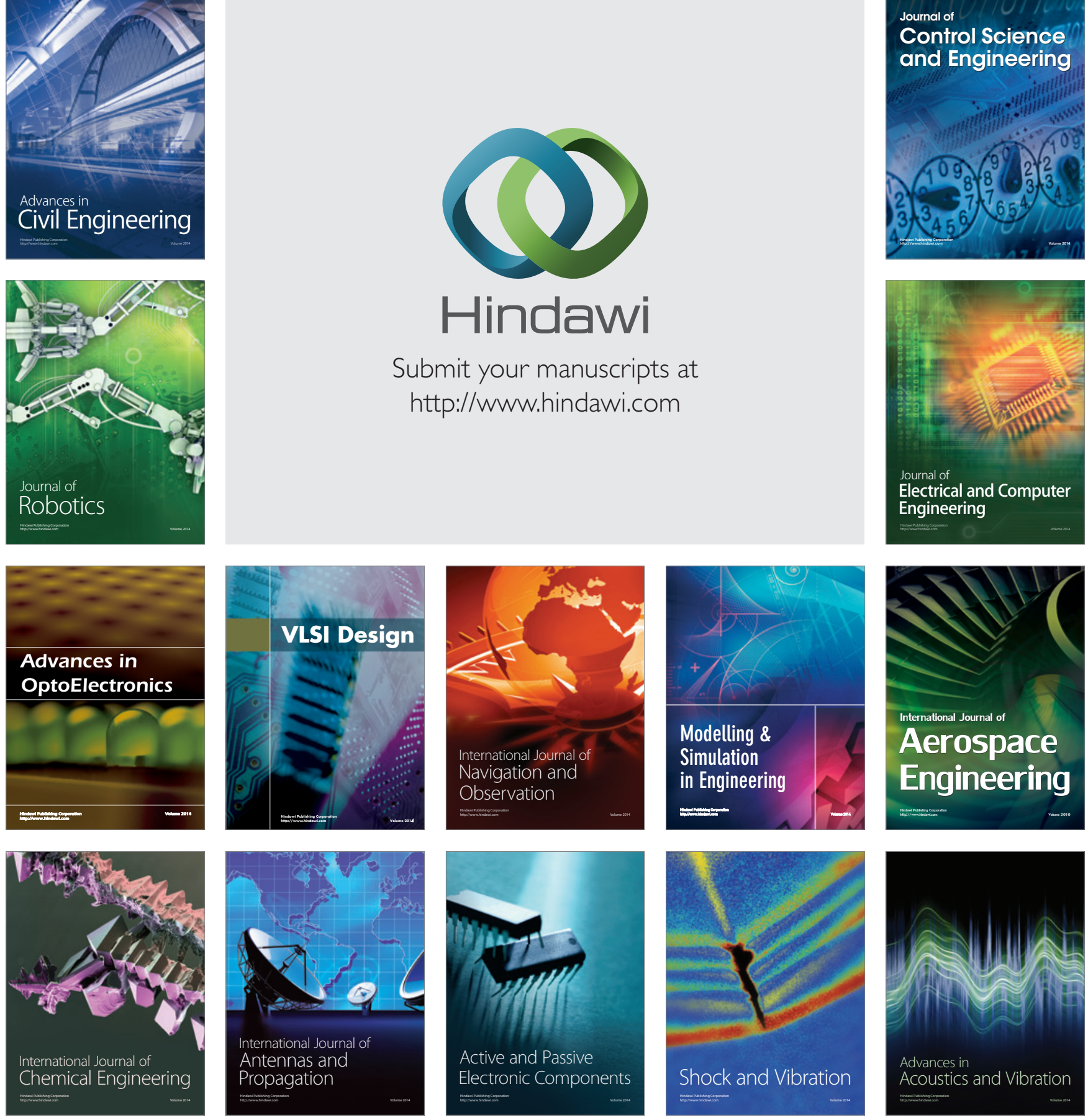